\title{
Pratiques
}

Linguistique, littérature, didactique

\section{Les enjeux de la didactique du français comme discipline de recherche et de formation}

\section{Claudine Garcia-Debanc}

\section{(2) OpenEdition}

\section{Journals}

Édition électronique

URL : http://journals.openedition.org/pratiques/1490

DOI : $10.4000 /$ pratiques. 1490

ISSN : 2425-2042

Éditeur

Centre de recherche sur les médiations (CREM)

Édition imprimée

Date de publication : 15 juin 2010

Pagination : 22-25

Référence électronique

Claudine Garcia-Debanc, «Les enjeux de la didactique du français comme discipline de recherche et de formation », Pratiques [En ligne], 145-146 | 2010, mis en ligne le 15 juin 2010, consulté le 30 avril 2019. URL : http://journals.openedition.org/pratiques/1490 ; DOI : 10.4000/pratiques.1490

(c) Tous droits réservés 


\section{Les enjeux de la didactique du français comme discipline de recherche et de formation}

La didactique du français langue première - cette appellation est désormais préférée à celle de didactique du français langue maternelle, dans la mesure où le français n'est pas toujours la langue maternelle des élèves qui sont scolarisés en France - est à la fois une discipline de recherche, une discipline impliquée dans la formation des enseignants et une discipline qui a des responsabilités sociales dans le champ du système scolaire.

Je tenterai de faire le point sur la situation actuelle de la didactique du français langue première comme discipline de recherche sous la forme de quelques remarques qui me paraissent dessiner un état des lieux de notre discipline.

Tout d'abord, on peut s'accorder sur le fait que la didactique du français langue première est une discipline scientifique jeune, dans la mesure où le terme n'est utilisé que depuis les années 1980, même si des travaux précurseurs, tel que le livre de Franck Marchand, Le Français tel qu'on l'enseigne ${ }^{(1)}$, publié en 1971, s'attachent déjà à décrire et analyser les pratiques d'enseignement en français et leurs effets sur les apprentissages des élèves. Elle se donne des objets d'étude originaux et se dote de méthodologies rigoureuses. Ainsi un des concepts-clés est celui de transposition didactique (voir le numéro 97-98 de la revue Pratiques, paru en juin 1998). La transposition didactique peut être définie comme une analyse des écarts entre les théories de référence et les contenus à enseigner, ce qui constitue le niveau 1 de la transposition didactique, selon Chevallard (1985-1991) ${ }^{(2)}$ ou transposition didactique stricto sensu, mais aussi des écarts entre les contenus à enseigner et les objets effectivement enseignés dans les classes, ce qui constitue le niveau 2 de la transposition didactique ou transposition interne, et enfin entre ce qui est enseigné et ce qui est appris par les élèves, niveau 3 de la transposition didactique. Les méthodes d'analyse sont différentes selon le niveau de la transposition didactique étudié : analyse de discours, notamment des programmes des manuels scolaires et des textes d'élèves pour les niveaux 1 et 3, linguistique interactionnelle pour interroger des transcriptions d'enregistrements vidéo pour le niveau 2. Historiquement, la didactique du français s'est successivement intéressée au niveau 1 , notamment avec les premiers articles de la revue Pratiques sur la diffusion des concepts de narratologie pour l'enseignement du récit au collège et au niveau 3 , l'analyse de copies d'élèves, avant de s'attacher à une étude des pratiques observées dans les classes. Cependant, les travaux de Marceline Laparra et ceux de Jean-François Halté ont très tôt porté sur les interactions orales dans la classe, comme en témoigne le numéro 103-104 de la Revue Pratiques (1999), intitulé Interactions et apprentissage, qui propose des analyses croisées d'une leçon de grammaire sur l'adverbe dans une classe de CM2. Le développement technologique des outils d'enregistrement, d'abord audio puis vidéo, a très probablement eu une influence non négligeable sur le développement de travaux relatifs au niveau 2 de la transposition didactique.

De même que pour les mathématiques, les Sciences Physiques ou l'EPS, la didactique du français est liée à la discipline scolaire correspondante et en étudie l'histoire. Ainsi André Chervel a pu montrer, dans son Histoire de l'enseignement du

(1) MARCHAND, F. (1971) : Le français tel qu'on l'enseigne, Paris : Larousse.

(2) Chevallard, Y. (1985-1991): La transposition didactique. Du savoir savant au savoir enseigné, Grenoble, La Pensée Sauvage. 
français du $X V I^{e}$ au $X X^{e}$ siècle, publiée en $2006^{(3)}$, l'évolution des enjeux assignés à l'enseignement du français, les modalités d'émergence d'une grammaire scolaire et les changements notables observés tout au long de cette période pour ce qui est des exercices et des critères d'évaluation. Contre la tentation de certains didacticiens d'autonomiser des didactiques relatives à chacun des objets d'enseignement particuliers (la didactique de la lecture littéraire, la didactique de l'orthographe, la didactique de l'écriture...), Jean-François Halté a montré la nécessité de prendre en compte la discipline scolaire français dans son ensemble. En effet, dès son ouvrage de la collection « que sais-je ? » consacré à la Didactique du français, publié en 1992, il propose le concept de " configuration didactique » pour interroger la cohérence d'ensemble de la discipline français à partir de « données de divers types, Instructions officielles, manuels scolaires, réformes, structures du système, rôle et fonction de la certification, discours issus de la noosphère...» (Halté, 1992, p. 21). L'analyse vise à mettre en évidence la solidarité entre les conceptions des finalités assignées à la discipline et la place relative accordée aux activités de pratique de la langue (oral, lecture, écriture) et aux activités d'étude de la langue (grammaire, orthographe, conjugaison, vocabulaire), la nature des corpus littéraires travaillés et des exercices ou activités dominants. L'étude porte principalement sur les prescriptions des programmes, les matériels d'enseignement et les revues professionnelles. Considérant l'action effective de l'enseignant dans la classe, le concept de « modèle disciplinaire en actes » proposé par Garcia-Debanc $(2004,2008,2009){ }^{(4)}$ tente de rendre compte des écarts entre les discours des enseignants et les pratiques observées dans leurs classes en interprétant les éléments intriqués qui apparaissent notamment au moment de la gestion des incidents critiques en classe comme tensions entre plusieurs modèles d'enseignement de la discipline historiquement situés. En effet, l'enseignant de français se réfère aux programmes en vigueur mais aussi aux programmes antérieurs, aux notions et pratiques qui ont été travaillées dans le cadre de sa formation initiale, aux références professionnelles des collègues de son établissement scolaire, voire à ses propres souvenirs d'élève. Les pratiques observées se trouvent donc influencées par toutes les strates des enjeux et des usages d'enseignement de la discipline.

En tant que discipline scientifique, la didactique du français langue première a elle-même une histoire. De la même manière que Francine Cicurel a proposé une histoire de la didactique du français langue étrangère à partir de l'analyse des articles parus dans une revue professionnelle, le Français dans le Monde, on pourrait envisager de construire une histoire de la didactique du français langue première en étudiant l'évolution des thématiques traitées par les principales revues du champ, Pratiques, Enjeux, Repères. On peut regretter la difficulté de diffusion de certains travaux fondateurs et l'amnésie de certains jeunes chercheurs en didactique du français langue première. Ainsi, par exemple, la question de la transversalité de la maîtrise de la langue dans les différentes disciplines scolaires n'est pas une question nouvelle mais

(3) Chervel, A. (2006) : Histoire de l'enseignement du français du XVI au XX $X^{e}$ siècle, Paris / Retz.

(4) Garcia-Debanc, C. (2004). «Les modèles disciplinaires en acte dans les pratiques effectives d'enseignants débutants ». Colloque international AIRDF, Québec, Août 2004. Actes.

GARCIA-DEBANC, C. (2008). « De la configuration didactique au modèle disciplinaire en acte : trente ans de didactique du français avec Jean-François Halté ». Pratiques 137-138, 39-56.

GARCIA-DEBANC, C. (2009). "Quand les enseignants débutants enseignent la relation sujet/verbe. De l'analyse didactique de pratiques observées de professeurs des écoles débutants à la détermination d'éléments d'expertise professionnelle pour l'enseignement grammatical », in Simard, Dolz (coord.) Les pratiques d'enseignement de la grammaire dans la francophonie, Presses Université Laval, pp. 99-124. 
a été explorée en didactique du français dès le début des années 80 . Dans cette perspective de réflexion épistémologique et de valorisation des travaux anciens, il est important de recenser et de mettre à la disposition des chercheurs les publications dans ce domaine, comme le font les banques internationales de documentation telles que la DAF.

La didactique du français langue première a une responsabilité dans le champ social. On peut se rappeler que, aux origines de la discipline, se trouvent le scandale que représente l'échec massif de certaines couches sociales et la lutte contre l'échec scolaire. La visée praxéologique de transformation des pratiques d'enseignement dans le sens d'une démocratisation de l'école, même si elle n'est pas unique et même si elle a été mise en question par certains chercheurs, notamment par Bernard Schneuwly dès le colloque de Poitiers en $2000^{(5)}$, se marque à plusieurs niveaux du système éducatif. Elle intervient dès la noosphère avec la participation de didacticiens à l'élaboration de certains programmes : Plan de Rénovation de l'enseignement du français en 1970 (même si, à l'époque, on ne parle pas encore de didactique mais de «pédagogie du français »), programmes français de l'école primaire de 2002 et 2007, programmes suisses du collège ou programmes québécois du collège et du lycée. Au cours des trente dernières années, les textes institutionnels de programmes scolaires se positionnent ainsi soit en convergence avec les résultats des recherches didactiques les plus récentes (ainsi par exemple de la présence de la réécriture dans les programmes de l'école primaire en France dès 1995 ou de l'introduction dans les programmes français pour l'école primaire de 2002 d'un enseignement de la littérature fondé sur les principes proposés par les recherches conduites à l'INRP ${ }^{(6)}$ dans ce domaine), soit en opposition à ceux-ci. De même, les recherches didactiques veillent à la diffusion des principaux résultats de leurs travaux dans la formation initiale et continue des enseignants. Les modes de diffusion des produits de la recherche didactique constituent en soi une question de recherche. Certains numéros de revues ont été consacrés à ce type de travaux, notamment le $n^{\circ} 13$ de la revue belge de didactique Diptyque, éditée par le Centre d'Etudes et de Documentation pour l'Enseignement du Français de l'Université de Namur, intitulé « Formation des enseignants et enseignement de la lecture et de l'écriture " ${ }^{(7)}$. J'ai essayé, dans une contribution à un ouvrage collectif paru en $2009^{(8)}$, de montrer l'évolution historique des relations entre recherches en didactique du français langue première et formation des enseignants, en focalisant l'étude sur les recherches sur l'écriture et la lecture : on est passé d'une visée transmissive et applicationniste et d'études de faisabilité reposant sur des innovations pédagogiques contrôlées à la prise en compte des contraintes du travail enseignant (Goigoux, $2002^{(9)}$ ) et à l'étude de la formation elle-même et de ses effets sur les pratiques enseignantes. On pourra trouver un échantillon de ce type d'études notamment dans le dossier de la Lettre de l'AIRDF $\mathrm{n}^{\circ} 43^{(10)}$ et dans un

(5) Marquillo, M. (coord.). Journées d'étude de la DFLM, Cahiers FORELL, Université de Poitiers.

(6) Institut National de Recherche Pédagogique.

(7) Dolz, J. \& Plane, S. (eds.). (2008). Formation des enseignants et enseignement de la lecture et de l'écriture. Recherches sur les pratiques, Namur.

(8) GARCIA-DEBANC, C. (2009). «L'articulation entre recherche en didactique du français et formation des enseignants du premier degré de 1970 à nos jours. Le cas de la production écrite », in Clanet J. (dir.) Recherche/formation des enseignants. Quelles articulations?, Rennes, Presses Universitaires de Rennes, 33-44.

(9) Goigoux, R. (2002). Analyser l'activité d'enseignement de la lecture : une monographie. In Revue Française de Pédagogie, 138, pp. 125-134.

(10) GARCIA-DEBANC, C. et DufAYS, J.-L. (2008). "Les pratiques enseignantes en français et leurs effets sur les apprentissages des élèves ». Dossier de la Lettre de l'AIRDF (Association Internationale des chercheurs et didactique du Français) n ${ }^{\circ} 43$ 2008-2, Bruxelles. 
ouvrage collectif rendant compte des travaux conduits au sein du GRIDIFE ${ }^{(11)}$ : dans ces recherches sont collectées et traitées des données relatives à la mise en œuvre d'action de formation dans divers domaines de l'enseignement du français (grammaire, lecture, écriture) et à leurs effets sur les pratiques observées des enseignants. Ainsi, depuis son origine, sous des modalités différentes, la perspective d'un usage social des résultats des recherches en didactique reste toujours importante.

De nombreuses directions de travail sont actuellement très fécondes. Ainsi tout d'abord de la confrontation avec les concepts-clés d'autres didactiques disciplinaires, lorsque la didactique du français langue première essaie d'éprouver la validité et les limites des concepts de «milieu » ou de « référence » pour rendre compte des apprentissages en français. La maturité scientifique de la discipline se signale par la collecte de données précises sur les pratiques observées, les recherches de ce type étant de plus en plus nombreuses. La collecte et l'analyse de grands corpus scolaires, oraux ou écrits, les études longitudinales sur les effets à court, moyen et long terme d'interventions d'enseignement, sont des directions de développement pour les prochaines années, dont l'exploitation pose les questions méthodologiques rencontrées aussi par les didactiques des autres disciplines : cadres théoriques à choisir, nature des corpus à collecter, grain d'analyse pour leur traitement.

Je voudrais enfin signaler quelques-uns des risques qui menacent actuellement la didactique du français langue première en tant que discipline scientifique. Nous avons déjà signalé le risque d'amnésie, lorsque les jeunes chercheurs en didactique $\mathrm{du}$ français langue première n'ont pas accès aux travaux pionniers de cette discipline. L'institutionnalisation universitaire progressive des enseignements en didactique du français langue première présente un grand nombre d'avantages pour la formation méthodologique des chercheurs mais fait aussi courir le risque d'académisme, y compris dans la formation initiale des maîtres. Dans le domaine de la formation continue des enseignants, le terme de didactique étant souvent utilisé comme synonyme de «relatif à l'enseignement », le risque est grand d'une dilution scientifique et méthodologique. De plus, certains éléments de didactique professionnelle mal comprise peuvent entrainer une insuffisante prise en compte de la spécificité des contenus à enseigner et enseignés.

La mise en regard des pratiques observées et des effets sur les apprentissages des élèves est centrale pour la didactique du français langue première. Mais ce travail ne fait que commencer. Il est pourtant indispensable, pour que le didactique du français langue première occupe toute sa place dans le champ social, moins sous la forme du militantisme de l'époque pionnière que par la mise à disposition des chercheurs, des décideurs politiques, de la communauté enseignante et des formateurs d'enseignants de données issues de recherches rigoureuses pouvant aider à une prise de décision informée dans des domaines aussi cruciaux que les premiers apprentissages en lectureécriture, l'enseignement de la lecture, de l'écriture ou de la grammaire.

Claudine Garcia-Debanc, IUFM Midi-Pyrénées,

Ecole interne de 1'Université Toulouse2-Le Mirail, CLLE-ERSS, UMR 5263 \& GRIDIFE-ERT 64

(11) Carnus, M-F, Garcia-Debanc, C., Terrisse, A. (2008). Analyse des pratiques des enseignants débutants : approche didactique, Grenoble, La Pensée Sauvage Editions. 